\title{
Not guilty-TMPRSS2-ERG does not sensitize cells to radiation
}

Approximately half of prostate cancers harbour the TMPRSS2$E R G$ gene fusion, which leads to ERG (v-ets erythroblastosis virus E26 oncogene homolog [avian]) overexpression. As this particular genetic lesion requires a double-strand DNA break to occur, a long held view is that tumours harbouring the fusion have inherently weak DNA repair capabilities, which would render them particularly sensitive to radiotherapy. New research published in Clinical Cancer Research has disproved this hypothesis.

"We used two methods to measure the fusion in the prostate cancer samples: array comparative genomic hybridization and immunohistochemical (IHC) staining for the ERG protein," explains lead investigator Robert Bristow. Pretreatment biopsy specimens from $>200$ patients with intermediate-risk prostate cancer (T1-T2, Gleason score $<8$ and serum PSA levels $<20 \mathrm{ng} / \mathrm{ml}$ ) were analysed. As a group, these men have variable outcomes, with a considerable proportion not responding to treatment, regardless of which modality they receive. In this study, patients underwent imageguided radiotherapy with a median dose of $76 \mathrm{~Gy}$.

On multivariate analysis, TMPRSS2-ERG status was not a prognostic factor for biochemical relapse-free rate. These results mirrored earlier findings that fusion status was not prognostic for biochemical recurrence after radical prostatectomy, which is unsurprising given that surgery does not introduce DNA damage.

"We found that the fusionpositive tumours are not more radiosensitive than the fusionnegative tumours." Bristow continues, "This result suggests that caution should be used in attributing fusion status as a marker of DNA repair status in patients with prostate cancer. Indeed, in cancer research, it is just as important to show the nonutility of a biomarker as the utility so the field can move forward."

Future studies might identify a biomarker (or panel thereof) that can predict radiotherapy treatment. "We are completing whole-genome sequencing on the patients in this study as part of the Canadian Prostate Cancer Genome Network sequencing project to develop an unbiased signature for radiotherapy failure or success," says Bristow.

These and other ongoing efforts should contribute to the development of precision treatments for patients based on their inherent tumour genetics.

Mina Razzak

Original article Dal Pra, A. et al. TMPRSS-ERG status is not prognostic following prostate cancer radiotherapy: implications for fusion status and DSB repair. Clin. Cancer Res. doi:10.1158/1078-0432.CCR-13-1049

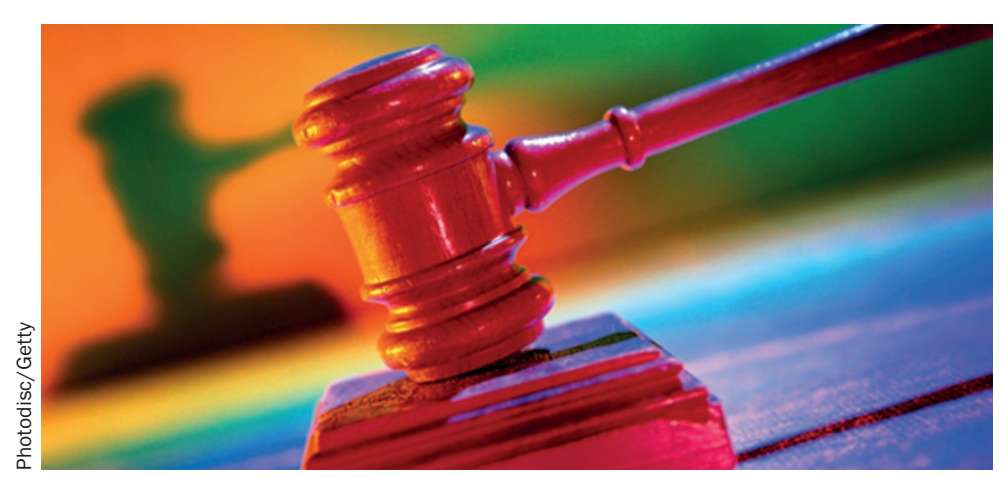

